\title{
TOPOLOGY OF NEGATIVELY CURVED REAL AFFINE ALGEBRAIC SURFACES
}

\author{
CHRIS CONNELL AND MOHAMMAD GHOMI
}

\begin{abstract}
We find a quartic example of a smooth embedded negatively curved surface in $\mathbf{R}^{3}$ homeomorphic to a doubly punctured torus. This constitutes an explicit solution to Hadamard's problem on constructing complete surfaces with negative curvature and Euler characteristic in $\mathbf{R}^{3}$. Further we show that our solution has the optimal degree of algebraic complexity via a topological classification for smooth cubic surfaces with a negatively curved component in $\mathbf{R}^{3}$ : any such component must either be topologically a plane or an annulus. In particular we prove that there exists no cubic solutions to Hadamard's problem.
\end{abstract}

\section{INTRODUCTION}

The classical examples of complete negatively curved surfaces in $\mathbf{R}^{3}$ are the hyperbolic paraboloid and the hyperboloid of one sheet, which have Euler characteristics 1 and 0 respectively. In 1898 Hadamard [17] studied the problem of constructing complete negatively curved surfaces with negative Euler characteristic, and described some qualitative semialgebraic solutions by merging hyperboloids of one sheet; see the Appendix. In this paper we obtain an explicit algebraic solution to Hadamard's problem. Specifically we show that the quartic equation

$$
3 z^{4}+2(1+4 x y) z^{2}-2\left(x^{2}+y^{2}\right)^{2}+8 x y-1=0,
$$

determines a smooth negatively curved surface $\Sigma \subset \mathbf{R}^{3}$ homeomorphic to the doubly punctured torus, which has Euler characteristic -2, see Figure 1. Hadamard's problem has been studied by a number of authors [2, 4, 28, 29], and in recent years there has been renewed interest in this problem due to applications in general relativity [7, 8]; however, explicit solutions have been very rare. Indeed, other than the example found here, we know of only one explicit solution to Hadamard's problem due to Vaigant [4, p. 55], which is an analytic quadruply punctured sphere.

We prove that $\Sigma$ is negatively curved (Sections 2 ) by a direct computation. Furthermore, we show that this quartic surface may be regarded as an optimal solution to Hadamard's problem by proving that there exists no smooth cubic surfaces in $\mathbf{R}^{3}$ with negative curvature and Euler characteristic (Section 3 ). The proof of this

Date: Last Typeset May 26, 2007.

1991 Mathematics Subject Classification. Primary 53A05, 51R10; Secondary 14P25, 51R12.

Key words and phrases. Negative Gaussian curvature, real algebraic surface, quartic, cubic.

The first named author was partially supported by NSF grant DMS-0420432. The second named author was supported in part by NSF CAREER award DMS-0332333. 


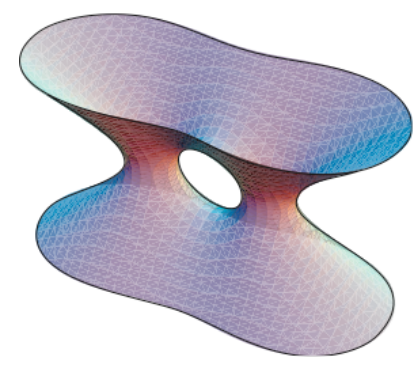

FIGURE 1

result uses the known classifications of cubics and their lines, specially the relatively recent work of Knörrer and Miller [20].

The algebraic properties of the nonsingular cubic surfaces and some of the singular cases have been thoroughly expounded in Segre's classic text [32]. Cayley [5] and Salmon [30] showed that there are always 27 lines on a nonsingular cubic in $\mathbf{C P}^{3}$. Much of the geometry surrounding the arrangements of these lines had been worked out by numerous authors in the mid 1800's. Among the many other mathematicians who took part in this effort are Clebsch [10, 11], Cremona [14], Gordan [9], Klein [19], and Steiner [33]. Around this time, Schläfli [31] and Cayley [6] classified the singular surfaces into various types, but not in terms of the modern language of singularity theory in the sense of Arnold [1]. This was carried out by Bruce and Wall [3] for the $\mathbf{C P}^{3}$ case and by Knörrer and Miller [20] in the $\mathbf{R P}^{3}$ case.

Note 1.1. There are many questions in mathematical literature which are referred to as "Hadamard's problem". We emphasize that by Hadamard's problem throughout this paper we mean constructing examples of complete negatively curved surfaces with negative Euler characteristics embedded in $\mathbf{R}^{3}$. In particular, this is not to be confused with "Hadamard's conjecture" on the existence of bounded negatively curved surfaces, see Note 1.4 below.

Note 1.2. We obtained (1) by experimenting with Cassini ovals, which are the locus of points in the plane whose product of distances from a pair of fixed points are constant. Specifically, note that if we set

$$
\bar{x}:=\frac{\sqrt{2} x}{\sqrt{1+z^{2}}}, \quad \bar{y}:=\frac{\sqrt{2} y}{\sqrt{1+z^{2}}}, \quad \bar{z}:=\frac{\sqrt{2} z}{\sqrt{1+z^{2}}},
$$

then (1) may be rewritten as

$$
\left((\bar{x}-1)^{2}+(\bar{y}-1)^{2}\right)\left((\bar{x}+1)^{2}+(\bar{y}+1)^{2}\right)=1+2 \bar{z}^{2}
$$

So each horizontal cross section of $\Sigma$ is a rescaling of a Cassini oval about the points $\pm(1,1)$, see Figure 2. In particular note that these curves never become convex as $z$ grows large. This is not accidental. Indeed convex cross sections of an algebraic surface would have to be strictly convex, and if a compact negatively curved surface is bounded by a pair of strictly convex curves in parallel planes, then the Gauss map of the surface is one-to-one on each boundary component; therefore, since the 

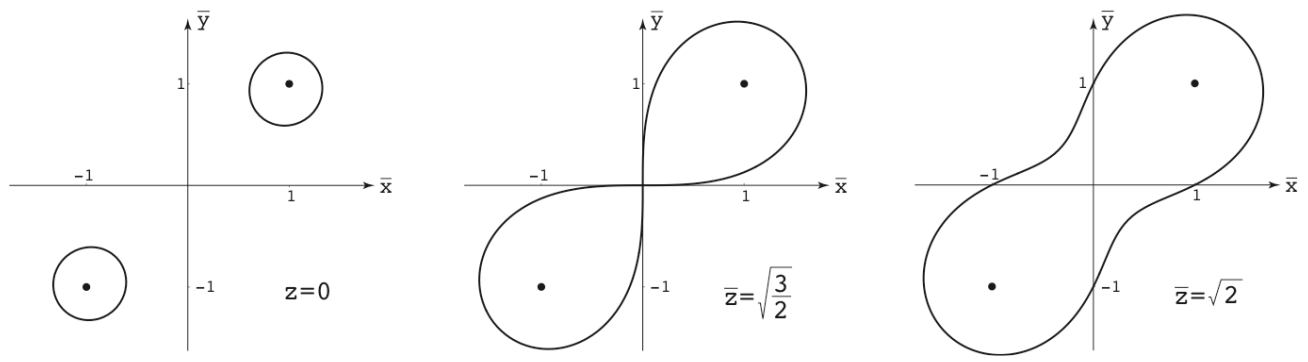

Figure 2

Gauss map is locally one-to-one everywhere (due to the nonvanishing of curvature) it would follow that the gauss map is a homeomorphism onto its image [15], which would force the surface to be homeomorphic to an annulus.

Note 1.3. There have been a host of complete minimal surfaces with negative Euler characteristic (and finite topology) discovered in recent years. Since these surfaces are minimal, they necessarily have nonpositive Gaussian curvature; however, none of these surfaces are known to have everywhere negative curvature. Indeed Meeks and Perez have conjectured [25] that a complete embedded minimal surface in $\mathbf{R}^{3}$ with negative curvature is either a catenoid, a helicoid, or a scherk 1- or 2-periodic surface; in particular, there exists no minimal examples of complete embedded negatively curved surfaces with negative Euler characteristic in $\mathbf{R}^{3}$.

Note 1.4. It is easy to construct examples of complete embedded negatively curved surfaces in $\mathbf{R}^{3}$ with nonnegative Euler characteristic, which are contained in between a pair of parallel planes. Consider for instance the semialgebraic surfaces

$$
y=x z+\frac{z^{n}}{1-z^{2}}, \quad-1<z<1 ; \quad \text { and } \quad x^{2}+y^{2}=\frac{1}{1-z^{2}}, \quad-1<z<1 .
$$

Figures 3(a) and (b) depict the first example for $n=1$ and 0 respectively, and Figure 3(c) depicts the second example. On the other hand, we do not know of any

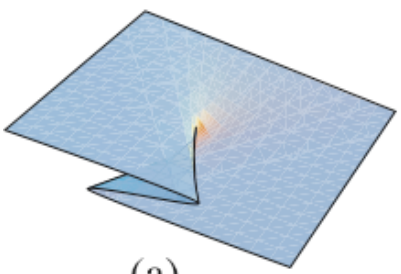

(a)

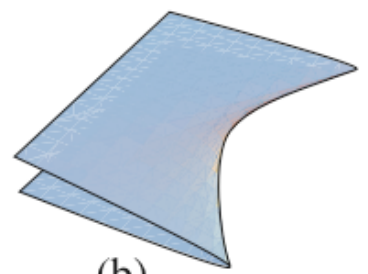

(b)

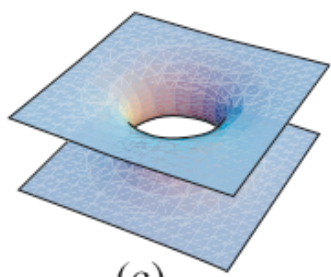

(c)

FiguRE 3

examples of complete negatively curved surfaces with negative Euler characteristic in a slab. In particular, it is not possible to merge two copies of the surface in 3(c) in the same manner that Hadamard merged two hyperboloids (see the Appendix), because in this case the curve of intersection between the two surfaces changes 
concavity. We should also mention that a conjecture attributed to Hadamard states that there exists no complete embedded surfaces of negative curvature in a ball in $\mathbf{R}^{3}[4,29]$. In the immersed case, this conjecture was settled by Nadirashvili [26] who constructed complete minimal negatively curved surfaces in a ball, see also $[18,13,23,22]$; however, such surfaces cannot be embedded, since by a recent result of Colding and Minicozzi [12], an embedded minimal surface must be unbounded.

Note 1.5. How could one construct other explicit solutions to Hadamard's problem, i.e., with topologies different from that of $\Sigma$ ? One way might be to experiment with generalized Cassini ovals, i.e., the locus of points whose product of distances from three or more points are constant (see Note 1.1). One may also wonder if it is possible to obtain new topological solutions by applying projective transformations to $\Sigma$. To achieve this one would "blow up" a plane which interesects $\Sigma$ in a singular curve. For instance the transformation $(x, y, z) \mapsto(x / z, y / z, 1 / z)$ blows up the $x y$ plane. Figure 4 shows three projective transformations of $\Sigma$ obtained in this way, see [16] for explicit formulas for these surfaces. Any projective transformation of $\Sigma$

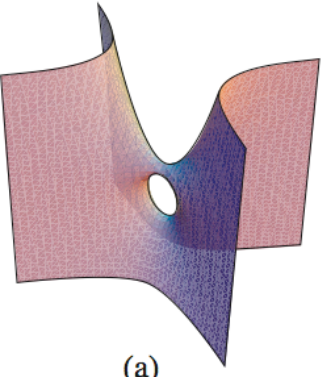

(a)

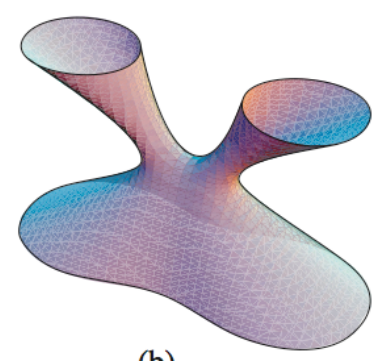

(b)

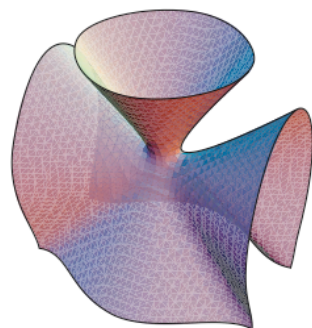

(c)

FiguRE 4

will necessarily have nonpositive curvature everywhere; however, all the surfaces in Figure 4 also have exactly 4 flat points along the plane which was blown up. Furthermore, these points are not colinear. Consequently no projective transformation of $\Sigma$ which changes its topology may have everywhere negative curvature, because any such surface could also be obtained by a projective transformation of, say, the surface (a), but there is only one plane which contains all flat points of surface (a) and blowing that one up returns $\Sigma$.

We should also mention that recently some examples of smooth immersed surfaces of nonpositive curvature with only cross-cap cusps have been constructed ([27]) by smoothing singular "hedgehog" (hérisson) surfaces based on the example of Martinez-Maure [24]. However, so far no nonsingular examples have been produced with strictly negative curvature.

\section{Proof that the Curvature of $\Sigma$ is Everywhere Negative}

There are two main cases to consider: 
2.1. $\mathbf{z} \neq \mathbf{0}$. By symmetry we may assume that $z>0$. Solving (1) for $z$ and taking its positive real root, we find that $\Sigma \cap\{z>0\}$ is the graph of $f(x, y) / \sqrt{3}$ where

$$
f(x, y):=\sqrt{-1-4 x y+\sqrt{2\left(2+3 x^{4}-8 x y+14 x^{2} y^{2}+3 y^{4}\right)}} .
$$

The sign of the curvature of $f$ is determined by the sign of

$$
\operatorname{det} \operatorname{Hess} f(x, y)=\frac{-2\left(p(x, y)+q(x, y) \sqrt{4+6 x^{4}-16 x y+28 x^{2} y^{2}+6 y^{4}}\right)}{\left(3 x^{4}+14 x^{2} y^{2}+3 y^{4}-8 x y+2\right)^{2}\left(\sqrt{4+6 x^{4}-16 x}-1-4 x y\right)^{2}},
$$

where

$$
\begin{aligned}
& p(x, y)=\left(670 x^{4} y^{4}-1424 x^{3} y^{3}+900 x^{2} y^{2}-176 x y+16\right) \\
&+\left(60 x^{2} y^{2}-168 x y+114\right)\left(x^{4}+y^{4}\right)+45\left(x^{8}+y^{8}\right),
\end{aligned}
$$

and

$$
q(x, y)=2(2 x y-1)\left(15\left(x^{2}-y^{2}\right)^{2}+20 x^{2} y^{2}+28 x y-4\right) .
$$

One may use a computer to quickly check that the numerator of $\operatorname{det} \operatorname{Hess} f$ is negative, which is all we need, see Note 2.1 below. We include, however, a verifiable and transparent proof of this by considering the following three subcases. In each case the problem is reduced to checking the sign of some polynomials of degree at most 4. The computer notebook [16] contains all these computations.

2.1.1. $\mathbf{x y} \geq \mathbf{1} / \mathbf{2}$. It is enough to check that $q>0$ and $p>0$. This is immediate for $q$. For $p$, note that if $x y \geq 1 / 2$, then $\left(x^{8}+y^{8}\right) \geq \frac{1}{2}\left(x^{4}+y^{4}\right)-2 x^{4} y^{4}$. So $45\left(x^{8}+y^{8}\right) \geq 10\left(x^{8}+y^{8}\right)+70 x^{4} y^{4} \geq 5\left(x^{4}+y^{4}\right)+50 x^{4} y^{4}$, which in turn yields the following lower bound for $p(x, y)$ :

$$
\left(720 x^{4} y^{4}-1424 x^{3} y^{3}+900 x^{2} y^{2}-176 x y+16\right)+\left(60 x^{2} y^{2}-168 x y+119\right)\left(x^{4}+y^{4}\right) .
$$

Neither of these polynomials in $x y$ have real roots. So it follows that $p>0$.

2.1.2. $\mathbf{0} \leq \mathbf{x y} \leq \mathbf{1} / \mathbf{2}$. If $\operatorname{det}$ Hess $f$ vanishes, then so does

$$
g(x, y):=p^{2}(x, y)-q^{2}(x, y)\left(4+6 x^{4}-16 x y+28 x^{2} y^{2}+6 y^{4}\right) .
$$

Further note that det Hess $f(0,0)<0$. So we just need to show that $g(x, y) \neq 0$ when $0 \leq x y \leq 1 / 2$ and $x$ and $y$ are not both zero. To this end we compute that

$g(x, y)=27\left(x^{2}+y^{2}\right)^{2}\left(g_{1}(x y)+g_{2}(x y)\left(x^{4}+y^{4}\right)+g_{3}(x y)\left(x^{8}+y^{8}\right)+75\left(x^{12}+y^{12}\right)\right)$, where $g_{1}(x), g_{2}(x)$, and $g_{3}(x)$ are given respectively by

$$
\left(40000 x^{4}-33409 x^{3}+13304 x^{2}-2496 x+192\right)+\left(3900 x^{3}-24160 x^{2}+1976 x+1\right) x^{3},
$$

$1125 x^{4}-4160 x^{3}+4272 x^{2}-2112 x+508$, and $-750 x^{2}+240 x+180$. It suffices to check that these polynomials are all positive on $[0,1 / 2]$, which is indeed the case. 
2.1.3. $\mathbf{x y}<\mathbf{0}$. Again we show that $g(x, y)$ does not vanish. Note that

$$
g(x, c x)=27\left(c^{2}+1\right)^{2} x^{4} \sum_{i=0}^{6} a_{2 i}(c) x^{2 i}
$$

where $a_{0}(c)=192, a_{2}(c)=-2496 c, a_{4}(c)=4\left(127 c^{4}+3326 c^{2}+127\right)$, and

$$
\begin{aligned}
a_{6}(c) & =-192 c\left(11 c^{4}+174 c^{2}+11\right), \\
a_{8}(c) & =12\left(15 c^{8}+356 c^{6}+3498 c^{4}+356 c^{2}+15\right), \\
a_{10}(c) & =80 c\left(c^{4}-22 c^{2}+1\right)\left(3 c^{4}+14 c^{2}+3\right), \\
a_{12}(c) & =75\left(c^{6}-5 c^{4}-5 c^{2}+1\right)^{2} .
\end{aligned}
$$

By symmetry it is enough to show that $\sum_{i=0}^{6} a_{2 i}(c) x^{2 i}>0$ when $x>0$ and $-1 \leq$ $c<0$. When $c<0$ all $a_{2 i}$, except $a_{10}$, are clearly positive. Further it is easy to verify that $a_{10}+a_{12}>0$ and $a_{8}+a_{10}>0$ on $[-1,0]$, since these are quartics in terms of $c^{2}$. Thus when $x \geq 1, a_{10} x^{10}+a_{12} x^{12} \geq\left(a_{10}+a_{12}\right) x^{10}>0$, and when $0 \leq x \leq 1, a_{8} x^{8}+a_{10} x^{10} \geq\left(a_{8}+a_{10}\right) x^{10}>0$ for all $-1 \leq c<0$. So, for $x \geq 0$ and $-1 \leq c<0$, we have $\sum_{i=0}^{6} a_{2 i}(c) x^{2 i} \geq a_{8}(c) x^{8}+a_{10}(c) x^{10}+a_{12}(c) x^{12}>0$.

2.2. $\mathbf{z}=\mathbf{0}$. After the substitution $(x, y) \longmapsto(x-y, x+y) / \sqrt{2}$, (1) becomes

$$
z^{4}+2\left(1+2 x^{2}-2 y^{2}\right) z^{2}=2\left(x^{2}+y^{2}\right)^{2}-4 x^{2}-4 y^{2}+1
$$

and $\Sigma$ becomes symmetric with respect to all coordinate planes. There are two subcases to consider:

2.2.1. $\mathbf{y} \neq \mathbf{0}$. By symmetry, we may assume that $y>0$. Solving (3) for $y$ and taking its positive real root we set

$$
f(x, z):=\sqrt{-1-x^{2}-z^{2}+\sqrt{\left(5 z^{4}+8 x^{2} z^{2}+6 z^{2}+8 x^{2}+1\right) / 2}} .
$$

Then the portion of $\Sigma$ which intersects the $x y$-plane in the interior of the first quadrant is the graph of $y=f(x, 0)$. A basic computation shows that

$$
\operatorname{det} \operatorname{Hess} f(x, 0)=\frac{-96 x^{4}-208 x^{2}-42+4\left(12 x^{2}+7\right) \sqrt{16 x^{2}+2}}{\left(8 x^{2}+1\right)^{2}\left(-2 x^{2}+\sqrt{16 x^{2}+2}-2\right)^{2}} .
$$

The above expression vanishes only when the following expression is zero:

$$
\left(-96 x^{4}-208 x^{2}-42\right)^{2}-16\left(12 x^{2}+7\right)^{2}\left(16 x^{2}+2\right) .
$$

But this is a quartic in terms of $x^{2}$ with no real roots.

2.2.2. $\mathbf{y}=\mathbf{0}$. It remains to check the curvature of $\Sigma$ where it intersects the $x$-axis, which is at 4 points. By symmetry we may assume that $x>0$, so there remain only two points. To find these we solve (3) for $x$ and taking its positive real roots, we set

$$
f_{ \pm}(y, z):=\sqrt{1-y^{2}+z^{2} \pm \sqrt{\left(5 z^{4}-8 y^{2} z^{2}+6 z^{2}-8 y^{2}+1\right) / 2}} .
$$

The points where $\Sigma$ intersects the positive portion of the $x$-axis are $\left(f_{ \pm}(0,0), 0,0\right)$, and straight forward computations show that det Hess $f_{ \pm}(0,0)=-7<0$. 
Note 2.1. Some procedures in Mathematica, including "Reduce", appear to quickly verify that the numerator of det Hess $f$ in Section 2.1 is negative, see [16]. According to $[34$, p. 1070], "Reduce" uses cylindrical algebraic decomposition for real domains via Gröbner basis, the Collins-Hong algorithm, and Strzebonski's genealogy-based method using validated numerics backed up by exact algebraic number computation.

\section{Topological Classification of Smooth Cubic Surfaces with a Negatively Curved Component in $\mathbf{R}^{3}$}

The simplest example of a negatively curved cubic in $\mathbf{R}^{3}$ is perhaps $z=x y+y^{3}$, which is topologically a plane. Next consider $z\left(x^{2}+y^{2}-z^{2}-1\right)=1$, which is obtained by perturbing the union of a hyperboloid of one sheet and the $x y$-plane. This surface is smooth and it has two components: a topological plane containing some positive curvature, and a topological cylinder which is everywhere negatively curved. The main result of this section is that these are topologically the only possibilities for a negatively curved component of an affine cubic:

Theorem 3.1. If a connected component of a smooth cubic surface in $\mathbf{R}^{3}$ is negatively curved, then it is homeomorphic to a plane or a cylinder.

We will break the proof of Theorem 3.1 into two cases depending on whether or not the smooth cubic in $\mathbf{R}^{3}$ has at most isolated singularities "at infinity." A precise description for this term is as follows. If we denote the (homogeneous) coordinates in $\mathbf{R P}^{3}$ by the equivalence classes $[x: y: z: w], \mathbf{R}^{3}$ may be naturally identified with the plane $w=1$. Then for any set $A \subset \mathbf{R}^{3}$, we denote its closure in $\mathbf{R P}^{3}$ with respect to the manifold topology by $\bar{A} \subset \mathbf{R P}^{3}$. Conversely, whenever we denote a closed set in $\mathbf{R} \mathbf{P}^{3}$ by $\bar{A}$, then $A:=\bar{A} \cap \mathbf{R}^{3}$ will denote its restriction in $\mathbf{R}^{3}$. Similarly $\bar{\partial} A$ denotes the topological boundary of the set $A$ (not $\bar{A}$ ) in $\mathbf{R P}^{3}$ and $\partial A$ denotes its boundary in $\mathbf{R}^{3}$. We define the plane at infinity, which we denote as $\mathbf{R} \mathbf{P}_{\infty}^{2}$, as the set $\{w=0\}$. (We think of $\mathbf{R P}_{\infty}^{2}$ as a "sphere at infinity" with its antipodal points identified, and we may visualize $\mathbf{R P}^{3}$ as $\mathbf{R}^{3}$ plus this sphere with its identification.) Points of $\bar{A}$ at infinity refers to the set $\bar{A} \cap \mathbf{R} \mathbf{P}_{\infty}^{2}$.

Note 3.2. To see why the proof of Theorem 3.1 is nontrivial, we first note that every real affine cubic has a noncompact component, and thus a component without a topological obstruction to having negative curvature everywhere. Further, there are many cubic surfaces, such as the famous Clebsch diagonal, which are nonsingular in $\mathbf{R}^{3}$ and have negative Euler characteristic. More precisely, the space $\mathcal{T}$ of all real cubic surfaces in $\mathbf{R}^{3}$ or $\mathbf{R} \mathbf{P}^{3}$ is 19 dimensional. Within this space there are 5 connected components of surfaces which are nonsingular in $\mathbf{R P}^{3}$. The remaining singular surfaces can be decomposed into strata by a variety of criteria. We may regard $\mathcal{T}$ as the space of equivalence classes under scalar multiplication of all homogeneous degree three real polynomials in four variables. The 15-dimensional projective group, $\operatorname{PGL}(4, \mathbf{R})$, acts on $\mathcal{T}$ by the change of variables $(x, y, z, w) \mapsto A .(x, y, z, w)$ for any matrix representative $A$ of the class $[A] \in \operatorname{PGL}(4, \mathbf{R})$. If we represent $\mathcal{T}$ as all scalar equivalence classes of real cubic polynomials in three variables, then 
$[A] \in \mathrm{PGL}(4, \mathbf{R})$ acts on $\mathcal{T}$ by the change of variables

$$
(x, y, z) \mapsto\left(\frac{A_{1} \cdot(x, y, z, 1)}{A_{4} \cdot(x, y, z, 1)}, \frac{A_{1} \cdot(x, y, z, 1)}{A_{4} \cdot(x, y, z, 1)}, \frac{A_{1} \cdot(x, y, z, 1)}{A_{4} \cdot(x, y, z, 1)}\right)
$$

followed with multiplication by $\left(A_{4} \cdot(x, y, z, 1)\right)^{3}$. (Here $A_{i}$ means the i-th row of $A$.) This group preserves the sign of curvature and smoothness in the affine portion of the surface, except possibly along the portion of the image of $\mathbf{R P}_{\infty}^{2}$ in $\mathbf{R}^{3}$. This image corresponds to the hyperplane defined by $A_{4}^{-1} \cdot(x, y, z, 1)=0$ where $A^{-1}$ is the matrix inverse of $A$. In particular, negative curvature and smoothness are preserved by the subgroup of $\operatorname{PGL}(4, \mathbf{R})$ where the first three coordinates of $A_{4}$ vanish, which is just the affine group $\operatorname{GL}(3, \mathbf{R}) \ltimes \mathbf{R}^{3}$. Note that due to symmetries, not all PGL $(4, \mathbf{R})$ orbits are 15 -dimensional.

In principal, we could start with a section of $\mathcal{T}$ over the 4-dimensional moduli space $\mathcal{M}=\mathcal{T} / \operatorname{PGL}(4, \mathbf{R})$, such as the one given by Sylvester's canonical pentahedral representation, and then vary the $\mathbf{R P}_{\infty}^{2}$ to obtain the 7-dimensional orbit space of the affine group. However, a computational proof of Theorem 3.1 on this entire orbit space seems impractical due to the presence of many orbits of surfaces which are negatively curved. Indeed, even if we are handed a rational parametrization of optimal degree for a subfamily of such surfaces, determining the existence of real roots of the numerator of the curvature function in terms of the original coefficients of the cubic is a difficult endeavor since it will typically be of at least degree 8 in both parameters. Nevertheless, we will need to use a fairly direct method to rule out a few special subsets of orbits where the computations turn out to be relatively simple. For the vast majority of cases we will be able to employ the rich structural features found in cubic surfaces.

3.1. Surfaces with at most isolated singularities on $\mathbf{R P}_{\infty}^{2}$. The proof in this case proceeds by first deriving a pair of geometric lemmas which give obstructions to the existence of negative curvature everywhere on a surface. Following that, we exploit the arrangement of lines on singular cubic surfaces described by the relatively recent classification in [20] to satisfy the hypotheses of the lemmas. This allows us to rule out each candidate for the Hadamard's problem.

Recall that a curve in a surface in $\mathbf{R}^{3}$ is an asymptotic curve if its normal curvature is identically 0 (i.e., the second fundamental form for the surface vanishes along the tangent direction of the curve).

Lemma 3.3. Let $\Sigma \subset \mathbf{R}^{3}$ be a $\mathcal{C}^{2}$-immersed orientable surface with everywhere negative Gaussian curvature. Suppose there exists a piecewise differentiable simple closed curve $C \subset \Sigma$ with $n$ segments each of which is an asymptotic curve. Then $n$ is even.

Proof. Since $\Sigma$ has negative curvature, at each point $p \in \Sigma$ there exists a pair of distinct asymptotic lines $\ell_{1}(p), \ell_{2}(p) \in T_{p} \Sigma$, which, since $\Sigma$ is orientable, are globally well-defined, i.e., each is a continuous line field on $\Sigma$. For instance, we may let $\ell_{1}$ be the asymptotic line such that when it is rotated "clockwise" about $p$ it first enters 
the sectors of $T_{p} M$, determined by $\ell_{1}$ and $\ell_{2}$, where the directional curvatures of $\Sigma$ are positive, and let $\ell_{2}$ be the other asymptotic direction.

Now fix an orientation for $C$, let $C_{i}, i=1, \ldots, n$ denote the segments of $C$ enumerated consecutively, and set $C_{n+1}:=C_{1}$. Since $C_{i}$ are asymptotic curves, either $\ell_{1}$ or $\ell_{2}$ (but not both) must be tangent to each $C_{i}$. Further, since $C_{i}$ and $C_{i+1}$ meet each other transversely, if $\ell_{1}$ is tangent to $C_{i}$ then $\ell_{2}$ must be tangent to $C_{i+1}$ and vice versa. Thus, since $\ell_{1}$ and $\ell_{2}$ are continuous and $C$ is closed, $n$ must be even.

Note 3.4. The doubly ruled quadric surface of negative curvature $z-x y=0$ has closed piecewise linear asymptotic curves of every positive even number of pieces.

Since smooth cubic surfaces in $\mathbf{R}^{3}$ have no boundary, they are always orientable. Moreover, straight lines in any surface are always asymptotic curves which implies the following restriction.

Corollary 3.5. If $\Sigma$ contains a triangle or a pentagon, then it is not everywhere negatively curved.

Note 3.6. If a smooth oriented surface $\Sigma$ embedded in $\mathbf{R}^{3}$ contains a compact region $B \subset \Sigma$ bounded by a union of simple closed piecewise linear curves $C_{1}, \ldots, C_{k}$, then each $C_{i}$ is also intrinsically piecewise geodesic. In that case, the Gauss-Bonnet theorem states that

$$
2 \pi \chi(B)=\Theta+\int_{B} K d \sigma
$$

where $\Theta=\sum_{i=1}^{k} \sum_{j=1}^{n_{i}} \theta_{i j}$ is the sum of the signed exterior angles between all the intersecting pairs of line segments and $d \sigma$ is the area form. In particular, if $k=1$ and $C_{1}$ is planar then $\Theta=2 \pi$ and so $\chi(B)$ is at most 0 when $\Sigma$ is negatively curved. The next result will show that such a bounded region cannot arise, even in some noncompact cases.

Definition 3.7. A plane $\bar{P} \subset \mathbf{R P}^{3}$ is a bounding plane for a connected component $\Sigma$ of a surface in $\mathbf{R}^{3}$ if

(i) there is a component $B$ of $\Sigma-P$ such that $\bar{\partial} B \subset \bar{P}$ and

(ii) either $\bar{B}$ misses a plane $\bar{Q} \subset \mathbf{R P}^{3}$ distinct from $\bar{P}$, or $\bar{P} \neq \mathbf{R P}_{\infty}^{2}$ and $\sup _{x \in B} d_{\mathbf{R}^{3}}(x, P)$ is achieved at some point of $B$.

We will slightly abuse terminology and call any such component $B$ a bounded component of $\Sigma-P$, and is by definition a connected component of $\bar{S}-\bar{P}$.

An Eckardt point of a surface is where three asymptotic curves meet tangent to 3 distinct directions. (N.B. for real algebraic surfaces, an Eckardt point is usually defined to be a real point in the intersection of three lines, two of which may be (nonreal) conjugate complex lines. In our definition, the lines must be real.)

Lemma 3.8. If any connected component $\Sigma$ of a smooth affine algebraic surface contains an Eckardt point or admits a bounding plane, then it is not everywhere negatively curved. In the latter case, $\Sigma$ contains an open set of nonnegative curvature. 
Proof. At an Eckardt point the second fundamental form is identically 0 as there can be at most two co-isotropic directions for a nonzero two dimensional quadratic form. Since the ambient curvature of $\mathbf{R}^{3}$ vanishes, the Gauss equations imply that the Gauss curvature of $\Sigma$ at $p$ vanishes.

For the second statement, let $\bar{P} \subset \mathbf{R P}^{3}$ be the bounding plane of $\Sigma$, and let $B$ and $\bar{Q}$ be the surface and plane provided by the definition of bounding plane. First, we treat the case where $\bar{P}=\mathbf{R P}_{\infty}^{2}$.

By assumption, $B$ lies to one side of $Q$ in $\mathbf{R}^{3}$. Let $c:[0, \infty) \rightarrow \mathbf{R}^{3}$ be a unit speed ray normal to $Q$ with $c(0) \in Q$, and lying on the same side of $Q$ as $B$. Let $Q_{t}$ be the unique plane parallel to $Q$ passing through $c(t)$. Then there is a least upper bound $t_{0}$ for the set of all $t>0$ such that $Q_{t}$ is disjoint from $B$. By compactness, the plane $\bar{Q}_{t_{0}}$ intersects $\bar{B}$. However, $\bar{Q}_{t_{0}} \cap \mathbf{R} \mathbf{P}_{\infty}^{2}=\bar{Q} \cap \mathbf{R} \mathbf{P}_{\infty}^{2}$ which was assumed to be disjoint from $\bar{B} \cap \mathbf{R P}_{\infty}^{2}$. Therefore $Q_{t_{0}}$ intersects $B$ and is tangent to $B$ at each point of intersection, since $B$ lies entirely in a closed half space determined by $Q_{t_{0}}$. In particular, at each intersection point $x$, the second fundamental form of $B$ is positive semi-definite. Hence $B$ is not positively curved at $x$.

Now suppose $\bar{P} \neq \mathbf{R P}_{\infty}^{2}$. If $\sup _{z \in B} d(z, P)=d(x, P)$ for $x \in B$, then the plane $P^{\prime}$ parallel to $P$ at distance $d(x, P)$ on the same side of $P$ as $x$ intersects $B$ at $x$. Moreover $B-P^{\prime}$ lies entirely to one side of $P^{\prime}$ and $x$ does not lie in $\partial B \subset P$ since by assumption $B$ cannot be planar. Therefore $P^{\prime}$ is tangent at $x$ and $B$ has nonnegative curvature at $x$.

Suppose instead that there is a plane $\bar{Q}$ distinct from $\bar{P}$. Let $T \in \operatorname{PGL}(4, \mathbf{R})$ be any projective transformation that carries $\bar{P}$ to $\mathbf{R P}_{\infty}^{2}$. Since $B$ misses $\bar{P}$ and $\bar{Q} \neq \bar{P}, T(B)$ will still lie in $\mathbf{R}^{3}$ and $T(\bar{Q})$ will be a plane which is not $\mathbf{R P}_{\infty}^{2}$. By the first case, there is a point $T(x) \in T(B)$ with nonnegative curvature. Since $T$ preserves the sign of the curvature, $x \in B$ is not positively curved.

For the final statement, we note that if $B$ has positive curvature at $x$, then it has positive curvature in a neighborhood of $x$. If $B$ has zero curvature at $x$ then since $B-P^{\prime}$ lies to one side of $P^{\prime}$, either there is a neighborhood of $x$ containing an open set with positive curvature, or else the curvature identically vanishes on $B$. However, there cannot be an open set where the curvature vanishes since $B$ cannot be planar.

Note 3.9. Each of the conditions in the definition of a bounding plane is necessary for Lemma 3.8 to hold. In item (i), $\bar{\partial} B$ must belong to a single plane $\bar{P}$. For instance, a component $\Sigma$ of the affine quartic $\left(1-z^{2}\right)\left(x^{2}+y^{2}\right)-1=0$ is a smooth negatively curved cylinder of revolution bounded between two parallel affine planes $z=1$ and $z=-1$. If we take $B$ to be the component of $\Sigma-(\{x=0\} \cup\{y=0\})$ intersecting the positive orthant, then $\bar{B}$ misses the plane $\{x+y=0\}$ in $\mathbf{R P}^{3}$.

In item (ii) we must insist that $\bar{Q}$ and not just $Q$ be disjoint from $\bar{P}$. For if $B$ is the negatively curved cylindrical component of $z\left(x^{2}+y^{2}-1-z^{2}\right)-1=0$, then $B$ misses the affine plane $Q=\{z=0\}$. (However, $\bar{B}$ intersects every plane of $\mathbf{R P}^{3}$.)

Also, there are bounding planes which satisfy each case in item (ii) to the exclusion of the other. First, if $\Sigma=\left\{z=x^{2}+y^{2}\right\}$ and $P=\{x=0\}$, then $B=\{x<0\} \cap \Sigma$ has $\bar{\partial} B \subset \bar{P}$. We can set $\bar{Q}=\{z=-1\}$, while $\sup _{x \in B} d(x, P)=\infty$. On the other 
hand, for $B=\Sigma=\left\{z\left(x^{2}+y^{2}\right)=1\right\}$ and $P=\{z=0\}$, the maximum distance on $B$ to $P$ is achieved at the point $(0,0,1) \in B$ but every plane in $\mathbf{R P}^{3}$ intersects $\bar{B} \cap \mathbf{R} \mathbf{P}_{\infty}^{2}$ since this is the line $\{w=0=z\} \subset \mathbf{R} \mathbf{P}_{\infty}^{2}$.

Lastly, note that if $\bar{B}$ misses a line $\ell$ of $\mathbf{R P}_{\infty}^{2}$ and $B$ approaches $\bar{B} \cap \mathbf{R P}_{\infty}^{2}$ from one side only, then there is a plane $\bar{Q}$ missing $B$ with $\bar{Q} \cap \mathbf{R P}_{\infty}^{2}=\ell$. The second condition is necessary to exclude cases like the negatively curved hyperboloid $z^{2}=x^{2}+y^{2}-1$ which intersects every plane, but whose boundary in $\mathbf{R P}_{\infty}^{2}$ misses the line $\{z=0=w\}$.

The above lemma immediately implies the following.

Corollary 3.10. Let $\Sigma$ be a real affine component of a cubic surface $\bar{S} \subset \mathbf{R P}^{3}$ which possesses a bounding plane $P$, and a bounded component $B \subset \Sigma$. If $\Sigma^{\prime}$ is a negatively curved component of $S$ for a different choice of $\mathbf{R P}_{\infty}^{2}$, then an open set of $\bar{B}$ lies in a different component from $\Sigma^{\prime}$.

Now we may prove the main result of this subsection:

Proposition 3.11. Let $\Sigma$ be a negatively curved connected component of a cubic surface in $\mathbf{R}^{3}$. Suppose that $\Sigma$ has only isolated singularities at infinity. Then $\Sigma$ is homeomorphic to a plane or a cylinder.

Proof. All of the results we will need about the nonsingular real projective cubic surfaces, including the basic facts about singular ones, can be found in Segre's monogram [32]. We will use these facts to derive corresponding facts about the real affine surfaces. First, we let $\bar{S}$ represent the cubic surface in $\mathbf{R P}^{3}$ and let Sing be the set of singular points of $\bar{S}$. The number of components of $\bar{S}-$ Sing is at most two. One component always has closure with odd Euler characteristic, and is therefore nonorientable. If there are two components, then the closure of the other component is a weakly convex sphere in any affine subspace of $\mathbf{R P}^{3}$ containing it.

If $\Sigma$ is contained in a convex spherical component of $\bar{S}-\mathbf{S i n g}$, then $\Sigma$ can (topologically) consist of either a sphere or a disk corresponding to whether $\mathbf{R P}_{\infty}^{2} \cap$ $\bar{\Sigma}$ is the empty set, or either a point or a topological circle. Since the spherical component is weakly convex and Sing $\subset \mathbf{R P}_{\infty}^{2}$ by smoothness, these are the only possibilities. Therefore we may and will assume that $\Sigma$ lies in the component with odd Euler characteristic.

Segre also observed that the complement of all the lines in $\bar{S}$ is a collection of topological disks together with the possible spherical component. Hence the lines, together with their vertices of intersection, form the one skeleton of a cell decomposition for the nonorientable component of $\bar{S}$. In particular the configuration of lines can be used to compute the Euler characteristic. For each $p \in \mathbf{S i n g}$, let $\mu(p)$ denote the number of components of $U-\{p\}$ for any sufficiently small neighborhood $U$ of $p$ in $\bar{S}$. Define $\bar{Y}$ to be the unique minimal compactification of $\bar{S}-$ Sing resulting in a closed topological surface built by replacing each point $p \in$ Sing in $\bar{S}$ with $\mu(p)$ distinct points. The computation of $\chi(\bar{Y})$ has been carried out in the list of Figure 5. From that list we may also derive $\chi(\bar{S})$ in each case, by noting that $\chi(\bar{S})=\chi(\bar{Y})-\sum_{p \in \operatorname{Sing}}(\mu(p)-1)$. 
The next step is to relate $\chi(\Sigma)$ to $\chi(\bar{S})$. Since $\bar{S}$ has only isolated singularities, it does not have a planar component and so the plane $\mathbf{R P}_{\infty}^{2}$ intersects $\bar{S}$ in a cubic curve $\bar{C}$. For the moment, assume $\bar{S}-\operatorname{Sing}$, or equivalently $\bar{Y}$, has only one component. Let $n$ be the number of connected components of $\mathbf{R P}_{\infty}^{2}-\bar{C}$. By the classification of cubic curves, this consists of between one and four disks, or else up to one disk and an annulus. Let $s \in\{0,1\}$ be the number of annular components.

Note that all lines in $S$ are connected to each other through lines which meet $\mathbf{R P}_{\infty}^{2}$ from two sides. Hence there is at most one component of $S$ containing lines. This is also the only component of $S$ that can have negative Euler characteristic, and therefore when it exists, it must be our candidate for a counterexample $\Sigma$. Since singular points always belong to lines, we are obliged to make $\mathbf{R P}_{\infty}^{2}$ contain all singular points so that they do not reside in $\Sigma$.

Let $N$ be the number of connected components of $S$. Under the requirement that $\mathbf{R P}_{\infty}^{2}$ pass through all singularities of $\Sigma$, the number $N-n$ is invariant under equisingular isotopy. By checking each of the 45 cases, we see that $N-n \leq 1$ and $N-n \leq 0$ whenever $\bar{Y}$ does not contain a spherical component. Alternatively, Segre shows that every singular surface is a boundary point in the moduli space of one of the 5 components of smooth surfaces. Therefore we can verify the above inequalities for one surface in each of these 5 classes and derive the general statement from the lower semicontinuity of $N-n$ over degenerating isotopies.

The Euler characteristic satisfies $\chi(\bar{S})=\chi(S)+\chi(\bar{C})$. Since the components of $\mathbf{R P}_{\infty}^{2}-\bar{C}$ are disks $(\chi=1)$ and possibly an annulus $(\chi=0)$, it follows that $1=$ $\chi\left(\mathbf{R P}_{\infty}^{2}\right)=n-s+\chi(\bar{C})$. Since the Euler characteristic of a nonspherical component of $S$ is at most one we have, $\chi(S) \leq \chi(\Sigma)+N-1 \leq \chi(\Sigma)+n-1=\chi(\Sigma)-\chi(\bar{C})+s$ or simply $\chi(\Sigma) \geq \chi(\bar{S})-s \geq \chi(\bar{S})-1$.

Now suppose $\bar{Y}$ does have a spherical component. Let $\Sigma_{1}, \ldots, \Sigma_{i}$ be the components of $S$ corresponding to the spherical component of $\bar{Y}$. Since $N-n \leq 1$, we have $\chi(S) \leq \chi(\Sigma)+\sum_{j=1}^{i} \chi\left(\Sigma_{j}\right)+N-i-1 \leq \chi(\Sigma)+\sum_{j=1}^{i} \chi\left(\Sigma_{j}\right)+n-i=$ $\chi(\Sigma)+\sum_{j=1}^{i} \chi\left(\Sigma_{j}\right)+1-i-\chi(\bar{C})+s$ or $\chi(\Sigma) \geq \chi(\bar{S})-\sum_{j=1}^{i} \chi\left(\Sigma_{j}\right)+i-1-s$. Note from the table that whenever $\bar{Y}$ has two components, then $\chi(\bar{Y})=3$. Let Sing $_{0} \subset$ Sing be the subset of points belonging to the closure of both components of $\bar{S}$ - Sing. If $\mathbf{S i n g}_{0}$ is empty then $\chi(\bar{S})=3, i=1$ and $\chi\left(\Sigma_{1}\right)=2$ so that $\chi(\Sigma) \geq 3-2-s \geq 0$. If $\operatorname{Sing}_{0}$ contains only one point $p$ with $\mu(p)=2$, then $i=1$, $\chi(\bar{S})=3-1=2$ and $\chi\left(\Sigma_{1}\right)=2-1=1$ so $\chi(\Sigma) \geq 1-s \geq 0$. If $\mathbf{S i n g}_{0}$ contains two points each with $\mu(p)=2$, then $i=1$ and these points and the line segment between them belong to both $\bar{C}$ and $\bar{\Sigma}_{1}$ since singular points are always connected by lines and the spherical component is weakly convex. Therefore $\chi\left(\Sigma_{1}\right)=1, \chi(\bar{S})=1$ and $\chi(\Sigma) \geq-s$. Since we assumed that there are no singular line segments, the line segment between the two singular points does not belong to $\bar{\Sigma}$ since it belongs to $\bar{\Sigma}_{1}$. However, this segment does belong to $\bar{C}$ and the remaining portions of the line are a degeneration of a separating curve in $\mathbf{R P}_{\infty}^{2}$. Adding the segment turns the complementary annulus component into a disk in $\mathbf{R P}_{\infty}^{2}-\bar{C}$ so that $s=0$ and hence $\chi(\Sigma) \geq 0$. Combining the results so far, we may exclude from consideration 
cases $4,5,9,10,13,16,17,25,30,32,34,36,41,43,44$ and 45 since $\chi(\Sigma) \geq 0$ for each of these.

We refer the reader to the pictures in Figure 6 for a partial view of the arrangements of the lines in each equisingular isotopy class. In order to see the lines and intersections which are hidden from view, the reader may find it helpful to view the animated " $3 \mathrm{D}$ " version of each surface available on the web-site www. Cubics . AlgebraicSurface. net ([21]). In each picture, $\mathbf{R P}_{\infty}^{2}$ has been chosen so as to not contain any line nor singularity of the surface. However, the pictures do not always show all intersections between lines. To help identify triangles, we recall that any two lines intersecting at a point $p$ belong to a triangle unless either one of the lines passes through a singularity or else $p$ is an Eckardt point.

A consequence of the work of Schläfli is that within each equisingular isotopy class, the number of lines as well as which pairs of lines intersect is kept invariant. Since a plane intersects the surface in a cubic curve, no more than three lines can be coplanar. Also, all lines are tangent to the surface at a nonsingular intersection point, so any nonsingular multiple intersection point is an Eckardt point consisting of the intersection of exactly 3 coplanar lines. Through an isotopy, a line belonging to a triangle or an Eckardt point may undergo an elementary move whereby it either crosses, moves off of, or moves onto the intersection point of the other two lines all within the same plane. Therefore, if we let $\mathbf{G}(\bar{S})$ be the graph in $\mathbf{R P}^{3}$ whose vertices are the intersections of lines on $\bar{S}$ and whose edges are the line segments between intersection points, then for an equisingular isotopic surface $\bar{S}^{\prime}$ the graph $\mathbf{G}\left(\bar{S}^{\prime}\right)$ can be obtained from $\mathbf{G}(\bar{S})$ by a finite sequence of elementary moves. The elementary moves are also constrained by the fact that a line must be freely isotopic to its image within the surface, so that, for instance, it cannot jump across a hole. In general, equisingular isotopy classes with few lines do not usually have representatives admitting Eckardt points and thus have rigid cell decompositions.

The three lines forming any triangle in the surface form a total of 4 triangles in $\mathbf{R P}^{3}$ with the same vertices, which we call associate triangles. Therefore, the only way a triangle and its associates can be removed from any affine presentation of the surface is to place at least one vertex of the triangle into $\mathbf{R P}_{\infty}^{2}$. We also consider an Eckardt point and its three associated coplanar bigons with single vertex at the Eckardt point to be (degenerate) triangles as well. With this convention, the total number of triangles, and the triples of lines that form them, are held constant in each isotopy class. In the literature, a plane containing the three lines of a triangle or Eckardt point, is called a tritangent plane of the first or second kind. Noting that Eckardt points are degenerations of 2-cells, then the number of 2-cells plus Eckardt points is also invariant under isotopy. However, the number of edges as well as the incidence graph of polygonal 2-cells may vary through the isotopy.

The main point of the pictures is that, by revealing the cell structure of a single representative from each isotopy class, they determine the incidence relations between the pairs of lines and the position of the lines relative to the topology of the 
Liste 4. Topologische Typen reeller kubischer Flächen mit höchstens rationalen Doppelpunkten

\begin{tabular}{|c|c|c|c|c|}
\hline $\begin{array}{l}\text { Singularitäten- } \\
\text { konfiguration }\end{array}$ & $\begin{array}{l}\text { Realitäts- } \\
\text { index }\end{array}$ & $\begin{array}{l}\text { Anzahl der } \\
\text { reellen Geraden }\end{array}$ & $\begin{array}{l}\text { Eulerchar. } \\
\chi(\bar{Y})\end{array}$ & $\begin{array}{l}\text { Mögliche weitere } \\
\text { komplexe Singularitäten }\end{array}$ \\
\hline$\varnothing$ & 3 & 27 & -5 & \\
\hline$\varnothing$ & 2 & 15 & -3 & \\
\hline$\varnothing$ & 1 & 7 & -1 & $A_{1}, 2 A_{1}$ \\
\hline$\varnothing$ & 0 & 3 & 1 & $A_{1}, A_{2}$ \\
\hline$\varnothing$ & -1 & 3 & $1+2$ & $A_{1}, A_{2}$ \\
\hline$A_{1}^{-}$ & 3 & 21 & -3 & \\
\hline$A_{1}^{-}$ & 2 & 11 & -1 & \\
\hline$A_{1}^{-}$ & 1 & 5 & 1 & $A_{1}$ \\
\hline$A_{1}^{-}$ & 0 & 3 & $1+2$ & $A_{1}, A_{2}$ \\
\hline$A_{i}$ & 0 & 3 & 1 & $A_{1}, A_{2}$ \\
\hline $2 A_{1}^{-}$ & 3 & 16 & -1 & \\
\hline $2 A_{1}^{-}$ & 2 & 8 & 1 & \\
\hline $2 A_{1}^{-}$ & 1 & 4 & $1+2$ & $A_{1}$ \\
\hline$A_{2}^{\bar{*}}$ & 3 & 15 & -3 & \\
\hline$A_{2}^{-\mathrm{a}}$ & 2 & 7 & -1 & \\
\hline$A_{2}^{-}$ & 1 & 3 & 1 & \\
\hline$A_{2}^{+}$ & 0 & 3 & 1 & $A_{1}, A_{2}$ \\
\hline $3 A_{1}^{-}$ & 3 & 12 & 1 & \\
\hline $3 A_{1}^{-}$ & 3 & 12 & 1 & \\
\hline $3 A_{1}^{-}$ & 2 & 6 & $1+2$ & \\
\hline$A_{2}^{-} A_{1}^{-\mathrm{a}}$ & 3 & 11 & -1 & \\
\hline$A_{2}^{-} A_{1}^{-\mathrm{a}}$ & 2 & 5 & 1 & \\
\hline$A_{3}^{-}$ & 3 & 10 & -1 & \\
\hline$A_{3}^{-}$ & 2 & 4 & 1 & \\
\hline$A_{3}^{-}$ & 1 & 2 & $1+2$ & \\
\hline$A_{3}^{+}$ & 1 & 4 & 1 & $A_{1}$ \\
\hline $4 A_{1}^{-}$ & 3 & 9 & $1+2$ & \\
\hline$A_{2}^{-} 2 A_{1}^{-}$ & 3 & 8 & 1 & \\
\hline $2 A_{2}^{-}$ & 3 & 7 & -1 & \\
\hline $2 A_{2}^{-}$ & 2 & 3 & 1 & \\
\hline$A_{3}^{-} A_{1}^{-}$ & 3 & 7 & 1 & \\
\hline$A_{3}^{-} A_{1}^{-}$ & 2 & 3 & $1+2$ & \\
\hline$A_{4}^{-\mathrm{a}}$ & 3 & 6 & -1 & \\
\hline$A_{4}^{-\mathrm{a}}$ & 2 & 2 & 1 & \\
\hline$D_{4}^{-}$ & 3 & 6 & 1 & \\
\hline$D_{4}^{+}$ & 1 & 2 & 1 & \\
\hline $2 A_{2}^{-} A_{1}^{-}$ & 3 & 5 & 1 & \\
\hline$A_{3}^{-} 2 A_{1}^{-}$ & 3 & 5 & $1+2$ & \\
\hline$A_{4}^{-} A_{1}^{-\mathrm{a}}$ & 3 & 4 & 1 & \\
\hline$A_{5}^{-}$ & 3 & 3 & 1 & \\
\hline$A_{5}^{-}$ & 2 & 1 & $1+2$ & \\
\hline$D_{5}^{-}$ & 3 & 3 & 1 & \\
\hline $3 A_{2}^{-}$ & 3 & 3 & 1 & \\
\hline$A_{5}^{-} A_{1}^{-}$ & 3 & 2 & $1+2$ & \\
\hline$E_{6}^{-}$ & 3 & 1 & 1 & \\
\hline
\end{tabular}

a zerfällt in zwei äquisinguläre Isotopieklassen

FigurE 5. Classification of surfaces with isolated singularities with their line count and topological type from [20]. 

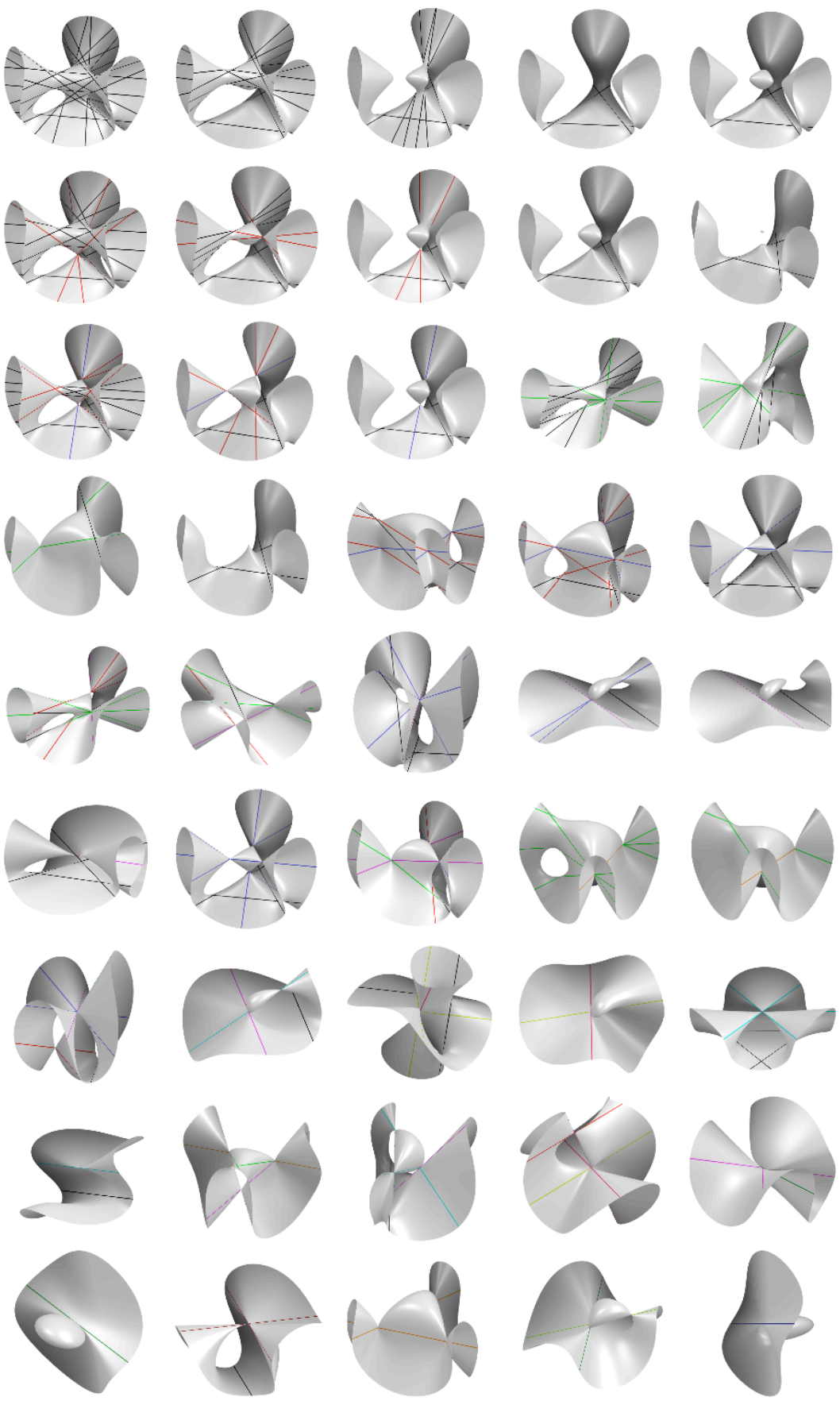

FiguRE 6. Arrangements of the lines on each of the 45 surfaces with at most isolated singularities. The multiplicity of lines passing through a singularity is indicated by the color. These are arranged in accord with the table, numbered across then down. (Courtesy of www.Cubics.AlgebraicSurface.net [21].) 
surface for all surfaces in each class. Nevertheless, we cannot appeal to the curvature of the representative nor to the placement of the $\mathbf{R P}_{\infty}^{2}$ as seen in the picture in order to rule out the whole equisingular isotopy class.

For what follows, if a triangle in $\bar{\Sigma}$, possibly with one or more vertices in $\mathbf{R P}_{\infty}^{2}$, lies in a bounding plane for $\Sigma$ and bounds a subsurface $B \subset \Sigma$, then we will call it a bounding triangle of $\Sigma$. A triangle or one or more of its associates may be a bounding triangle for $\Sigma$. For instance, if a triangle $T \subset \bar{\Sigma}$ separates $\bar{S}-T$ into two connected components such that $\mathbf{R P}_{\infty}^{2}$ misses at least one of these components, then $T$ is a bounding triangle for $\Sigma$. By Corollary 3.10, $\Sigma$ has an open set of nonnegative curvature if it contains a bounding triangle.

Suppose that, for some choice of $\mathbf{R P}_{\infty}^{2}$, say $\mathbf{R P}_{\infty}^{2}=\bar{Q}$, a plane $\bar{P}$ containing a triangle $\bar{T}$ in $\bar{S}$ is a bounding plane for a smooth component $\Sigma^{\prime}$ of $\bar{S}-\bar{Q}$ which contains $\bar{T}$. In that case, let $B_{1}, \ldots, B_{k}$ be the components of $\Sigma^{\prime}-\bar{P}$ which are bounded by $\bar{P}$ and note that each of them are the components bounded by $\bar{P}$. We will say that a plane $\bar{P}$ containing a triangle $T$ in $\bar{\Sigma}$ is obstructed if at least one vertex of $T$ belongs to $\mathbf{R P}_{\infty}^{2}$, and in the event that $P$ is a bounding plane for the component $\Sigma^{\prime}$ the component containing $T$ of some other affine realization $\bar{\Sigma}-\mathbf{R P}_{\infty}^{2}$ for some choice of $\mathbf{R P}_{\infty}^{2}$ whenever $T$ separates $\bar{S}$ into two components, a nonorientable one $B_{1}$ and an orientable one $B_{2}$, then $\mathbf{R P}_{\infty}^{2}$ either passes through a singular point in $B_{2}$ or else separates $B_{2}$ into at least two components one of which lies in a different component of $S$ from $\Sigma$. Note that if a plane $\bar{P}$ intersects $\bar{\Sigma}$ in a triangle, then $\bar{P} \cap \bar{\Sigma}$ contains precisely the three lines of the triangle since these form a maximal cubic curve. If $T$ is obstructed and lies in the plane $\bar{P} \neq \mathbf{R} \mathbf{P}_{\infty}^{2}$, then by Corollary 3.10, $\mathbf{R P}_{\infty}^{2}$ must intersect each component of $\bar{\Sigma}-\bar{P}$ in either a singular point or else a one dimensional curve.

Suppose $B_{1}, \ldots, B_{k}$ is a family of disjoint open smooth surfaces in $\bar{S}$ such that each $B_{i}$ is bounded by a triangle $T_{i} \subset \bar{\Sigma}$ with at least one vertex in $\Sigma$. Since any triangle $T$ misses a plane in $\mathbf{R P}^{3}$ distinct from the plane of $T$, it follows that for each $i, B_{i}-\mathbf{R} \mathbf{P}_{\infty}^{2}$ must consist of two nonempty components in $\mathbf{R}^{3}$. Since the $B_{i}$ are disjoint, this implies that for the cubic curve $\bar{C}=\mathbf{R P}_{\infty}^{2} \cap \bar{\Sigma}$, there are at least $k$ components of $\mathbf{R P}_{\infty}^{2}-\bar{C}$. If $\Sigma-\cup B_{i}$ has nonempty interior, then $\mathbf{R P}_{\infty}^{2}-\bar{C}$ has at least $k+1$ components. Since the maximum number of components of $\mathbf{R P}_{\infty}^{2}-\bar{C}$ for a cubic curve $\bar{C}$ is 2 if $\bar{C}$ contains no lines, 3 if it contains at least one line, and 4 if and only if it contains three lines in general position, this constraint makes obstructing triangles rather difficult.

If a triangle $T \subset \bar{\Sigma}$ is not obstructed then $\Sigma$ has nonnegative curvature by Corollary 3.5 and Lemma 3.8. The configuration of lines will allow us to rule out almost every case by finding simultaneously unobstructable triangles. In what follows we will always set $\bar{C}=\mathbf{R P}_{\infty}^{2} \cap \bar{S}$, and we will assume familiarity with the various possibilities for cubic curves in $\mathbf{R P}^{2}$.

1. This class is represented by the Clebsch diagonal cubic. Each such surface is smooth with the maximum number of real lines, 27 . It has 45 distinct planes containing triangles. A necessary condition for $\mathbf{R P}_{\infty}^{2}$ to obstruct all these triangles is that it contain one vertex from each one. Choose a linear 
projection of $\mathbf{R P}^{3}$ to a plane such that $\mathbf{R P}_{\infty}^{2}$ is sent to a line, $\ell$, and each line of the surface is sent to a line. Recall that no vertex of a triangle may be shared by another. So if $\ell$ is not the image of a line of the surface it must intersect the lines with total algebraic intersection number $45 * 2=90$, and more if there are Eckardt points. Since a line of a surface may belong to at most 5 triangle planes, if $\ell$ is the image of one or three lines of $\Sigma$, the only possibilities, then there are still at least $90-3 * 5-1=74$ intersection points counted with multiplicity. However, each of the images of the 27 lines can intersect $\ell$ at most once. Hence it is impossible to obstruct all triangles simultaneously.

2. This case has 15 lines and the same argument as in the first case applies. There are now 15 planes containing triangles. Projecting $\mathbf{R P}_{\infty}^{2}$ to $\ell$, there are at least either 30 (respectively, 25, or 14) intersection points counted with multiplicity if $\ell$ is the image of no (resp. 1 or 3 ) lines of $\Sigma$. However, there can be at most 15 (resp. 14 or 12) lines intersecting $\ell$.

3. Observe that there can be no Eckardt points in this case, so that the cell decomposition shown is rigid under isotopy. There is one line $\ell$ of $\Sigma$ that intersects all 6 of the remaining lines. If $\mathbf{R P}_{\infty}^{2}$ does not contain this line, then there is a unique plane that contains one vertex from each triangle. From the configuration we see that such a choice of $\mathbf{R P}_{\infty}^{2}$ must intersect $\ell$ between the innermost lines since no pair of these lines may cross in the isotopy. However, such a plane cannot obstruct the bounding triangle shown at the very bottom since it misses an interior component of one of its associated triangles. On the other hand, there is no choice of $\mathbf{R P}_{\infty}^{2}$ containing $\ell$ which obstructs both the topmost bounding triangle and the very bottom bounding triangle. To see this, recall that $\mathbf{R P}_{\infty}^{2}$ must intersect the surface in a cubic curve, so as it contains $\ell$ it can contain at most one other smooth parametrized subcurve, unless it contains two other lines. In particular, it misses one of the regions bounded by the two bounding triangles.

6. This has 21 lines. It also has 15 lines and 15 triangle planes which do not pass through the singular point. Therefore, the same argument as in case \#2 shows that no plane can contain a vertex of each triangle.

7. Here $\mathbf{R P}_{\infty}^{2}$ must create separate components from the disks bounded by the top central and middle central triangles each of which cannot degenerate since they possess a singular vertex. Additionally, $\mathbf{R P}_{\infty}^{2}$ must separate a component from the bottom central triangle, unless it degenerates to an Eckardt point in which case $\mathbf{R P}_{\infty}^{2}$ must pass through this point. In all cases, $\bar{C}$ is an impossible cubic curve.

8. None of the triangles can degenerate to Eckardt points since they would contain the singularity. Therefore there are always four disjoint disks bounded by bounding triangles. This makes $\bar{C}$ an impossible curve.

11. Any admissible choice of $\mathbf{R P}_{\infty}^{2}$ must contain both singular vertices, and it must also pass through the interior of the triangle shown at top center containing a singular vertex in order to obstruct it. Therefore, to obstruct 
the large lower nonsingular triangle, $\mathbf{R P}_{\infty}^{2}$ must pass through its vertex shown toward the back. (It must also pass through the intersection point shown front and center which is a vertex of a triangle sharing a line with the large lower central triangle.) In this case, $\mathbf{R P}_{\infty}^{2}$ intersects $\bar{S}$ in a cubic curve $\bar{C}$ of characteristic $\chi(\bar{C})=-2$. As shown, $\bar{S}-\bar{C}$ is connected. Since such a curve is maximal, $\mathbf{R P}_{\infty}^{2}$ cannot have any additional points of intersection with $\bar{S}$, and so $\bar{S}-\bar{C}$ is connected in the entire isotopy class. Hence $\chi(\Sigma)=$ $\chi(\bar{S})-\chi(\bar{C})=1$. (Alternatively $\mathbf{R P}_{\infty}^{2}$ cannot separate any of the disks bounded by triangles.)

12. $\mathbf{R P}_{\infty}^{2}$ must contain the line through the two singular points. However, there are also 3 disks bounded by triangles with a vertex at a singular point. (One having two singular vertices.) This makes $\bar{C}$ impossible.

14. We note that in order to obstruct the front and top most bounding triangle possessing a singular vertex $p$, it is necessary to choose $\mathbf{R} \mathbf{P}_{\infty}^{2}$ to cut the disk bounded by this triangle as well as contain $p$. Off of $p$, this disk intersection with $\mathbf{R P}_{\infty}^{2}$ belongs to a smooth subcurve of $\bar{C}$. There is a second triangle shown on the lower left with a vertex at the singular point and which shares one line, $\ell$, with the first triangle situated on top. This second triangle bounds a disk which therefore must also be intersected by $\mathbf{R P}_{\infty}^{2}$ since $\mathbf{R P}_{\infty}^{2}$ intersects $\ell$ only at the singular point, this intersection produces another smooth component in $\bar{C}-\{p\}$. However, there is a third bounding triangle with a vertex at the singular point on the right side of the picture as shown, symmetric to the second triangle with respect to the only reflection symmetry. If $\mathbf{R P}_{\infty}^{2}$ cuts the interior of the bounded disk of this triangle, then $\bar{C}-\{p\}$ contains yet another component. This cannot happen since $\bar{C}$ cannot reduce to three lines and still obstruct all the bounding triangles. These triangles persist in all representatives of the isotopy class since they contain $p$ and so a degeneration of a triangle to an Eckardt point at $p$ would cause another line to intersect $p$. (N.B. there are triangles on the hidden backside of the picture as shown which could conceivably degenerate to Eckardt points, so the entire cell complex is not necessarily invariant.)

15. There are three planes of triangles all sharing a common line, $\ell_{0}$, each containing one of the three mutually skew lines, $\ell_{1}, \ell_{2}$ and $\ell_{3}$, which are disjoint from the singular point. We will label these planes top, middle and bottom by their relative position along a vertical line through the midpoint of the picture. In the top triangle plane, two of the four associate triangles bound disks, $D_{1}$ shown at center top and $D_{2}$ shown front center and continuing on the back. Two of the triangles on the bottom plane span disks $D_{3}$, shown at top left and continuing on the bottom right, and $D_{4}$, shown at front right and continuing mostly hidden toward the left rear. The middle plane contains no bounding triangles. Therefore, $\mathbf{R P}_{\infty}^{2}$ must pass through the singularity as well as at least one point of the interior of each of the four bounded disks. However, if it passes through $D_{1}$ and $D_{4}$ then since it crosses $\ell_{0}$ at the singular point and $\ell_{3}$ on the $D_{4}$ side and not the $D_{3}$ side of $\ell_{0}$, 
$\mathbf{R P}_{\infty}^{2}$ is forced to intersect $D_{2}$ and $D_{4}$ in non intersecting components. The cubic curve $\bar{C}=\mathbf{R P}_{\infty}^{2} \cap \bar{\Sigma}$ must therefore have three components when the singular point is removed. This cannot happen unless $\bar{C}$ consists of three lines through the singular point. In that case it could not intersect any of the disks. Lastly note that the entire cell complex is combinatorially invariant under isotopy since none of the triangles can degenerate to an Eckardt point at the singularity because the lines $\ell_{1}, \ell_{2}$, and $\ell_{3}$ must remain nonsingular.

18. In this case there are three singular points so $\mathbf{R P}_{\infty}^{2}$ is determined and $\bar{C}$ consists of three lines. However, then the disk shown center top and bounded by a triangle cannot intersect $\mathbf{R P}_{\infty}^{2}$.

19. Here $\mathbf{R P}_{\infty}^{2}$ is again determined by the three singularities, but the topmost disk is disconnected. So instead we note that the disk shown top left and continuing on bottom right is bounded by a triangle and connected to $\Sigma$, but it cannot be intersected by $\mathbf{R P}_{\infty}^{2}$.

20. Use the same argument as in the previous two cases but note that the disk shown at center bottom is not intersected.

21. Both singular points must be in $\mathbf{R P}_{\infty}^{2}$ and it must cut the disk bounded by the triangle shown at top center in order to obstruct it. However, as in the previous two cases, the disk bounded by the triangle shown at lower front, and continuing at back right, with two singular vertices and sharing a line with the first triangle cannot be intersected by $\mathbf{R P}_{\infty}^{2}$.

22. $\mathbf{R P}_{\infty}^{2}$ must contain the line through the two singular points. However, it cannot then intersect both the disk shown at top center and the disk shown at lower right and continuing on the top left. Otherwise, $\bar{C}$ would have two disjoint ovals.

23. There are two triangle planes which do not pass through the singular point. We label the one shown at bottom as the first triangle and the one shown at left as the second. Note that they share a line, $\ell$, in common. Suppose the first such triangle shown at bottom does not degenerate to an Eckardt point in a given representative of the isotopy class. Since $\mathbf{R P}_{\infty}^{2}$ must contain an interior point of the disk it bounds, as well as the singularity, it must pass through the vertex of the second triangle which does not lie on $\ell$. On the other hand, if the first triangle degenerates to an Eckardt point, then either $\mathbf{R P}_{\infty}^{2}$ passes through the Eckardt point and the vertex of the second triangle not on $\ell$, or else $\mathbf{R P}^{2}$ intersects $\Sigma$ in exactly $\ell$ and the line of multiplicity 2 which passes through the singular point and intersects $\ell$. In all cases the cubic curve $\bar{C}=\mathbf{R P}_{\infty}^{2} \cap \Sigma$ has characteristic $\chi(\bar{C})=-1$. The complement $\bar{S}-\bar{C}$ is connected since it is connected for the given representative, and no new components can be created by maximality of $\bar{C}$. Hence $\chi(\Sigma)=\chi(\bar{S})-\chi(\bar{C})=0$.

24. There are two bounding planes which bound disks: one plane contains the three lines through the singular point, and the other contains the two parallel lines, as shown, only one of which passes through the singular point. Since 
$\mathbf{R P}_{\infty}^{2}-\bar{C}$ must have at least three components, $\chi(\bar{C}) \leq-2$ so $\chi(\Sigma)=$ $\chi(\bar{\Sigma})-\chi(\bar{C}) \geq 2$.

26. The $\mathbf{R P}_{\infty}^{2}$ must contain the singular point and one of the vertices of the lower triangle. However, there are two bounded disks of the plane of the lower triangle. Therefore $\mathbf{R P}_{\infty}^{2}-\bar{C}$ has at least three components and $\bar{C}$ has an additional isolated point. This is impossible for a cubic curve.

27. This is the unique class of surfaces with four singularities. The lines show that these are never coplanar, so there can be no smooth representatives.

28. This admits the same argument as case \#19.

29. $\mathbf{R P}_{\infty}^{2}$ must contain the line $\ell$ passing through both singularities. There are three planes containing triangles each sharing $\ell$ as a common line. However $\mathbf{R P}_{\infty}^{2}$ then cannot obstruct both the triangle with top-most remaining vertex and the one shown at bottom. Note that for each of these two triangles, two of the four associates bound disjoint disks, and the other two associates bound a common cylinder. Moreover, the entire cell complex is invariant under isotopy, since the singularities must be kept disjoint.

33. First note that there are three distinct planes containing triangles, all of which contain the singular point as a vertex. The singular point must be contained in $\mathbf{R P}_{\infty}^{2}$, which satisfies the vertex requirement for obstructing all three triangle planes. Only one of the three triangle planes, the one shown as approximately horizontal, contains a bounding triangle (shown with a vertex at infinity). In the plane of the bounding triangle, two of the four associate triangles bound a disk. Therefore $\mathbf{R P}_{\infty}^{2}-\bar{C}$ has at least three components. Hence $\chi(\bar{C}) \leq-2$, but for certain cases where $\chi(\bar{C})=-2$ it is possible for the curve to separate a disk component from each of the bounded disks. For instance, in the given picture, we may take $\mathbf{R P}_{\infty}^{2}$ to contain the horizontal line which passes through the singularity and which in the front passes very slightly above the line through the singularity shown extending from the front to the rear. In this case, all of our obstructions are satisfied since $\chi(\Sigma)=\chi(\bar{S})-\chi(\bar{C})+2=-1$, and we do not obtain the desired conclusion. By an argument similar to Lemma 3.8, it is possible to show that the closure of the disk separated by the triangle plane which is shown roughly in the plane of the paper has nonnegative curvature at a nonsingular point, even though this is not a bounding plane. However, this isotopy class has a relatively simple and small parameter space of normal forms which will allow us to rule it out easily by the direct argument given in Lemma 3.12 below.

35. The $\mathbf{R P}_{\infty}^{2}$ passes through the singularity and one of the vertices of the lower triangle. Since this triangle bounds a disk, there must be at least two components to $\mathbf{R P}_{\infty}^{2}-\bar{C}$. On the other hand, either $\bar{C}$ has a cusp or a line. In the first case no such curve exists. In the second case, since the curve contains an upper line and one of the lower vertices, it must also intersects in either another line or else a curve connecting the singular point to the lower vertex. In either case, $\chi(\bar{C})=-1$ with $\bar{S}-\bar{C}$ connected, or else it 
doesn't exist. (In fact no such curve can separate the disk bounded by the lower triangle to obstruct it.)

37. The argument of case \#18 applies here.

38. Here $\chi(\bar{\Sigma})=\chi(\bar{Y})-3=0$ and $\mathbf{R P}_{\infty}^{2}$ is determined uniquely by the condition that it contain all three singular points. Therefore $\chi(S)=\chi(\bar{S})-\chi(\bar{C})=3$, and $S$ has two disk components, so $\chi(\Sigma)=1$.

39. The two line containing the two singular points lies in $\mathbf{R P}_{\infty}^{2}$. No matter how $\bar{C}$ intersects $\bar{S}$ it must contain another portion of the curve incident on the $A_{4}$ singular point. Any cubic curves with a line incident to another smooth curve cannot have an isolated point or ellipse so there are no annulus components of $\mathbf{R P}_{\infty}^{2}-\bar{C}$. Hence $\chi(\Sigma) \geq \chi(\bar{S})=0$.

40. The tangent cone at the $A_{5}$ singular point consists of two planes intersecting in a line of the surface, one of which is a bounding plane containing the three lines of the surface. This plane bounds a disk shown at the left and continuing top right. Consequently $\mathbf{R P}_{\infty}^{2}$ must intersect this disk in a separating curve which also passes through the singular point. Therefore $\mathbf{R P}_{\infty}^{2}-\bar{C}$ has no annulus components which implies $\chi(\Sigma) \geq \chi(\bar{S})=0$.

42. This case is similar to \#40. The plane containing the two parallel lines bounds a disk which must be separated by $\mathbf{R P}_{\infty}^{2}$. Therefore the curve $\bar{C}$ meets the $D_{5}$ singularity with valence 4 , unless the entire disk is separated, in which case $\bar{C}$ consists of both parallel lines. In either case there can be no annulus component as $\bar{C}$ is connected. Hence $\chi(\Sigma) \geq 0$.

The next lemma will complete the proof of the proposition.

Lemma 3.12. Every component $\Sigma$ with negative Euler characteristic of a smooth affine cubic surface having an $A_{4}$ singularity in $\mathbf{R P}^{3}$, as in case \#33, has positive curvature somewhere.

Proof. We will use the classification in Section 2 of [3]. The $A_{4}$ singularity is a binode and falls under their Lemma 3 part (c) with $\left\{k_{0}, k_{1}\right\}=\{1,2\}$. This means that after a linear change of coordinates we may write the original cubic in homogeneous coordinates in the form $f(x, y, z, w)=x z w+f_{3}(x, y, w)$ where the cubic plane curve $f_{3}=0$ has a multiplicity 1 intersection with $w=0$ at the point $[x: y: w]=[0: 1: 0]$ and a multiplicity 2 intersection with the plane $x=0$ at $[0: 1: 0]$. Noting the discussion at the end of Case B of Section 2 in [3], if we place the singularity at the point $P=[0: 0: 1: 0]$, then after a linear change of coordinates we may write the cubic in homogeneous coordinates in the form

$$
f(x, y, z, w)=x z w+y w^{2}+x\left(a_{1} x^{2}+a_{2} x y+a_{3} y^{2}\right),
$$

where $a_{3} \neq 0$. Since projective transformations preserve the sign of curvature of the affine portion, we just need to check the curvature for all choices of $\mathbf{R P}_{\infty}^{2}$ containing the singularity. Keeping $P$ fixed and varying $\mathbf{R P}_{\infty}^{2}$ corresponds to replacing $w$ by $a_{4} x+a_{5} y+a_{6} w$, where $a_{6} \neq 0$. In affine coordinates, $\Sigma$ is therefore a component of the surface $S$ defined by $g=0$ where

$$
g(x, y, z)=z x\left(a_{4} x+a_{5} y+a_{6}\right)+y\left(a_{4} x+a_{5} y+a_{6}\right)^{2}+x\left(a_{1} x^{2}+a_{2} x y+a_{3} y^{2}\right) .
$$


Suppose $a_{5} \neq 0$. It is easy to check that the parametrized line $\ell(t)=\left(0,-\frac{a_{6}}{a_{5}}, t\right)$ belongs to $S$, and therefore it belongs to the candidate component $\Sigma$, since all lines are connected in $S$. We can compute,

$$
\operatorname{grad}(g)(\ell(t))=\left(\frac{a_{3} a_{6}^{2}}{a_{5}^{2}}, 0,0\right) .
$$

We conclude that the Gauss map along this line is constant so the Gauss curvature vanishes along $\ell$.

Now suppose $a_{5}=0$, then $\mathbf{R P}_{\infty}^{2}$ contains $\ell$. Solving for $z$ in $g=0$, we obtain

$$
z(x, y)=\frac{-y\left(a_{4} x+a_{6}\right)^{2}-x\left(a_{1} x^{2}+a_{2} x y+a_{3} y^{2}\right)}{x\left(a_{4} x+a_{6}\right)} .
$$

The components of $S$, if more than one, must be separated by the parallel planes $x=0$ and $a_{4} x+a_{6}=0$.

The curvature of $S$ is the Hessian of $z(x, y)$ divided by the square of the first fundamental form. Hence, if the Hessian is nonnegative at a point where $x\left(a_{4} x+\right.$ $\left.a_{5} y+a_{6}\right) \neq 0$, then the curvature is nonnegative there. The Hessian of $z(x, y)$ is easily computed to be,

$$
H=a_{6}^{2}\left(-\frac{a_{2}^{2}-4 a_{1} a_{3}}{\left(a_{6}+a_{4} x\right)^{4}}+\frac{2 x\left(a_{2} x+2 a_{3} y\right)}{x^{4}\left(a_{6}+a_{4} x\right)^{2}}-\frac{1}{x^{4}}\right) .
$$

Since $a_{3} \neq 0$ and $y$ occurs only in one term, for any $x \notin\left\{0,-\frac{a_{6}}{a_{4}}\right\}$, we can choose $y$ either sufficiently negative or sufficiently positive so that the expression for $H$ is positive. Hence each component of $S$ has positive curvature.

Therefore, in all cases, the curvature of any component with negative Euler characteristic is nonnegative somewhere.

Note 3.13. The underlying reason the curvature vanishes along $\ell(t)$ in the proof above, is that this line passes through the singular point with multiplicity three. It can be shown that any line that is a degeneration of three or more lines must have vanishing curvature along its entire length. We could have used this additional tool with the lemmas above to provide an alternate approach in ruling out each of the cases including \#33.

3.2. Other cases of singularities and the proof of Theorem 3.1. It remains to treat the cubics which are smooth in $\mathbf{R}^{3}$ but possess more than just isolated singularities at infinity. These remaining possibilities of singularities were classified in [20], and may be conveniently placed into the following three categories:

(1) Reducible surfaces, which consist of three planes or a plane and a possibly singular quadric.

(2) Irreducible ruled surfaces, which are not cones over an irreducible cubic curve in the plane. These are projectively equivalent (and equisingularly isotopic) to one of the following three surfaces represented in homogeneous 
coordinates with a solitary singular line at $0=w=x$ :

$$
\begin{aligned}
w^{2} y+x^{2} z & =0 \\
\left(w^{2}-x^{2}\right) y-2 w x z & =0 \\
x^{3}+w^{2} y+w x y & =0
\end{aligned}
$$

(3) An irreducible ruled surface which is a cone over an irreducible plane cubic. These are projectively equivalent to a surface described in homogeneous coordinates (in $\mathbf{R P}^{3}$ ) by

$$
x^{2} y-4 w^{3}+a w y^{2}-b y^{3}=0
$$

where the discriminant $a^{3}-27 b^{2}$ vanishes, i.e. $a=3|b|^{2 / 3}$, and the signs of $a$ and $b$ place the singularities into one of three types:

(a) $a \neq 0$ and $b>0$ (a line of self intersection along [0:2:z:1] if $b=1$ )

(b) $a \neq 0$ and $b<0$ (an isolated line along [0:-2:z:1] if $b=-1$ )

(c) $a=b=0($ cusp line at $[0: 1: z: 0])$

Note we have set the cone point at $[x: y: z: w]=[0: 0: 1: 0]$.

In the reducible case we either obtain three planes, or else a plane and a quadric. The planar cases all have 0 curvature, and they must be parallel, with possibly two or three planes identified, in order to be affinely smooth. For the case of a plane and quadric, the only way the plane has no singularities in $\mathbf{R}^{3}$ is to either add it as the plane at infinity, $w=0$, or else disjoint from the quadric. The latter case can occur when the quadric is an ellipsoid, paraboloid or a two-sheeted hyperboloid. In any case, the question reduces to which quadrics may have negative curvature, and these are always either planar (e.g. $z-x y=0$ ) or cylindrical (e.g. $x^{2}+y^{2}-1-z^{2}=0$ ) since a compact ellipsoid must always have positive curvature by Gauss-Bonnet, and no higher genus quadrics exist.

Lastly, in each of the ruled surface cases, if we place the singular line in the plane $w=0$, then each component in $\mathbf{R}^{3}$ is a plane. It is routine to verify this for each of the 6 explicit cubics above, where we set $b=1,-1,0$ in the irreducible conic case. This completes the proof of Theorem 3.1.

\section{Appendix: Sharp Estimate for Merging Two Hyperboloids}

Consider the hyperboloids of one sheet given by $F_{1}=0, F_{2}=0$ where

$$
\begin{aligned}
& F_{1}(x, y, z):=(x-2)^{2}+(y-2)^{2}+z^{2}-1 \\
& F_{2}(x, y, z):=(x+2)^{2}+(y+2)^{2}+z^{2}-1 .
\end{aligned}
$$

In $[17$, p. 42] Hadamard claims that

$$
F_{1} F_{2}=\epsilon, \quad F_{1}>0, \quad F_{2}>0,
$$

is a negatively curved surface, provided that $\epsilon$ is a rapidly decreasing function of the coordinates. Here we obtain a sharp estimate which shows that $\epsilon$ can be set equal to a constant. To this end we solve (4) for $z$ and take its positive real root to define

$$
f(x, y):=\sqrt{x^{2}+y^{2}-\sqrt{16(x+y)^{2}+\epsilon}+7} .
$$


The graph of $f$ is the portion of the solution to (4) in the half space $z>0$. As we have done earlier in this paper, we need to check the sign of

$$
\operatorname{det} \operatorname{Hess} f(x, y)=\frac{g\left((x+y)^{2}\right)}{\left(16(x+y)^{2}+\epsilon\right)^{3 / 2}\left(x^{2}+y^{2}-\sqrt{16(x+y)^{2}+\epsilon}+7\right)^{2}},
$$

where

$$
g(x)=-\epsilon^{2}-24 x \epsilon-112 \epsilon+\sqrt{16 x+\epsilon}(23 \epsilon-16 x) .
$$

We need to have $g(x)<0$ when $x \geq 0$. This is always the case when $x \geq 23 \epsilon / 16$. Further if $x<23 \epsilon / 16$, then

$$
g(x)<-\epsilon^{2}-24 x \epsilon-112 \epsilon+\sqrt{24 \epsilon}(23 \epsilon-16 x)<-\epsilon^{2}-112 \epsilon+46 \sqrt{6} \epsilon^{3 / 2} .
$$

The last expression vanishes only when $\epsilon=0$ or $\epsilon=6236 \pm 92 \sqrt{4593}$ and is negative between its two smallest roots. So it follows that $g(x)<0$ when

$$
0<\epsilon \leq 6236-92 \sqrt{4593} \approx 1.00585 .
$$

This yields that, for these values of $\epsilon$, the solution to (4) is negatively curved where $z>0$. By symmetry, the same estimate works in for $z<0$ as well. So it remains to consider where $z=0$. In this case we first substitute $(x, y) \mapsto(x-y, x+y) / \sqrt{2}$ so that our hyperboloids become symmetric with respect to the coordinate planes. Then we solve (4) for $y$ and take one of its positive real roots to obtain

$$
f(x, z):=\sqrt{-x^{2}+z^{2}+\sqrt{32 x^{2}+\epsilon}-7} .
$$

A computation shows that

$$
\operatorname{det} \operatorname{Hess} f(x, 0)=\frac{-\epsilon^{2}-48 \epsilon x^{2}-112 \epsilon+\sqrt{32 x^{2}+\epsilon}\left(23 \epsilon-32 x^{2}\right)}{\sqrt{\left(32 x^{2}+\epsilon\right)^{3}}\left(x^{2}-\sqrt{32 x^{2}+\epsilon}+7\right)^{2}} .
$$

Since the denominator of the last expression is always positive, we just need to check the sign of the numerator. To this end note that if $23 \epsilon-32 x^{2} \leq 0$, then the numerator is always negative. So suppose $23 \epsilon-32 x^{2}>0$. Then $x^{2}<23 \epsilon / 32$, and consequently the numerator is smaller than

$$
-\epsilon^{2}-48 \epsilon x^{2}-112 \epsilon+\sqrt{24 \epsilon}\left(23 \epsilon-32 x^{2}\right) \leq-\epsilon^{2}-112 \epsilon+46 \sqrt{6} \epsilon^{3 / 2},
$$

which, as we had computed before, is negative when (5) holds. Now it only remains to check the intersections with the $x$ axis. To this end we solve (4) for $x$, after the substitution $(x, y) \mapsto(x-y, x+y) / \sqrt{2}$, and take its positive real roots to define

$$
f_{ \pm}(y, z):=\sqrt{-y^{2}+z^{2} \pm \sqrt{32\left(1-y^{2}+z^{2}\right)+\epsilon}+9} .
$$

We then compute that

$$
\operatorname{det} \operatorname{Hess} f_{ \pm}(0,0)=\frac{\mp(\sqrt{\epsilon+32} \pm 16)^{2}}{(\epsilon+32)(\sqrt{\epsilon+32}-9)}
$$


These expressions are negative as long as $\epsilon<49$, which completes the proof that when (5) is satisfied, (4) has everywhere negative curvature. In particular, we conclude that the semialgebraic surface

$$
F_{1} F_{2}=1, \quad F_{1}>0, \quad F_{2}>0
$$

is negatively curved. This surface is depicted in Figure 7.

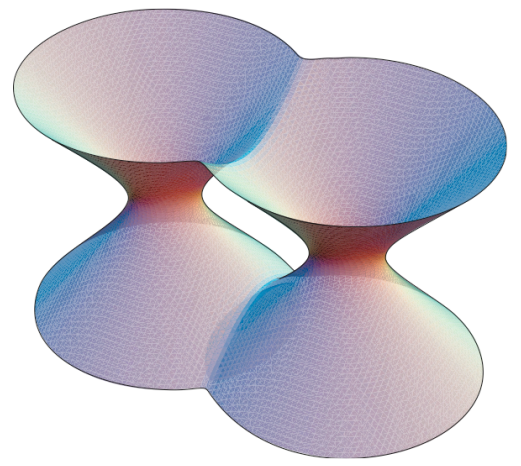

Figure 7

More generally it can be shown that for hyperboloids

$$
\begin{aligned}
& F_{1}(x, y, z):=(x-a)^{2}+(y-a)^{2}+z^{2}-r^{2}, \\
& F_{2}(x, y, z):=(x+a)^{2}+(y+a)^{2}+z^{2}-r^{2},
\end{aligned}
$$

the solution to (1) is negatively curved if and only if

$$
0<\epsilon \leq \frac{a^{2}}{r^{2}}\left(108 a^{4}-44 r^{2} a^{2}+7 r^{4}-\left|r^{2}-6 a^{2}\right| \sqrt{3\left(108 a^{4}-52 r^{2} a^{2}+11 r^{4}\right)}\right) .
$$

The argument is virtually identical to that given above for the case $a=2$ and $r=1$.

\section{REFERENCES}

[1] V. Arnold, Singularity theory, Development of mathematics 1950-2000, Birkhäuser, Basel, 2000, pp. 63-95.

[2] Bakel'man, I. Ya., Verner, A. L., and Kantor, B. E. Vvedenie v differentsialnuyu geometriyu " $v$ tselom" [Introduction to differential geometry "in the large"]. Izdat. "Nauka", Moscow, 1973.

[3] J. W. Bruce and C. T. C. Wall, On the classification of cubic surfaces, J. London Math. Soc. (2) 19 (1979), no. 2, 245-256.

[4] Y. D. Burago and S. Z. Shefel'. The geometry of surfaces in Euclidean spaces [ MR1039818 (91d:53004)]. In Geometry, III, volume 48 of Encyclopaedia Math. Sci., pages 1-85. Springer, Berlin, 1992.

[5] A. Cayley, On the triple tangent planes to a surface of the third order, Camb. and Dublin Math. Journal 4 (1849), 118-132.

[6] _ A memoir on cubic surfaces, Philos. Trans. Royal Soc. 159 (1869), 231-326.

[7] H. Chan. Embedding negatively curved initial data of black-hole collisions in $\mathbf{R}^{3}$. Classical Quantum Gravity, 23(1):225-234, 2006.

[8] H. Chan and A. Treibergs. Nonpositively curved surfaces in $\mathbf{R}^{3}$. J. Differential Geom., 57(3):389-407, 2001. 
[9] A. Clebsch and P. Gordan, Ueber die Theorie der ternären cubischen Formen, Math. Ann. 1 (1869), no. 1, 56-89.

[10] A. Clebsch, Über die Knotenpunkte der Hesseschen Fläche insbesondere bei Oberflächen dritter ordnung, J. Reine Angew. Math. 59 (1861), 193-228.

[11] _ Die Geometrie auf den Flächen dritter ordnung, J. Reine Angew. Math. 65 (1866), 359-380.

[12] T. H. Colding. and W. P. Minicozzi The calabi-yau conjectures for embedded surfaces. preprint.

[13] P. Collin and H. Rosenberg. Notes sur la démonstration de N. Nadirashvili des conjectures de Hadamard et Calabi-Yau. Bull. Sci. Math., 123(7):563-575, 1999.

[14] Cremona, Mémoire de géometrie pure sur les surfaces du troisième ordre, J. Reine Angew. Math. 68 (1868), 1-133.

[15] M. Ghomi. Gauss map, topology, and convexity of hypersurfaces with nonvanishing curvature. Topology, 41(1):107-117, 2002.

[16] _ NegativeCurvature.nb. Mathematica notebook, available at www.math.gatech.edu/ ghomi/MathematicaNBs.

[17] J. Hadamard. Les surfaces a courboures opposees et leurs lignes geodesiques. J. Math. Pures Appl., 4(1):27-73, 1898.

[18] L. P. d. M. Jorge and F. Xavier. A complete minimal surface in $\mathbf{R}^{3}$ between two parallel planes. Ann. of Math. (2), 112(1):203-206, 1980.

[19] _ Über Flächen dritter ordnung, Math. Annalen 6 (1873), 551-581.

[20] H. Knörrer and T. Miller, Topologische Typen reeller kubischer Flächen, Math. Z. 195 (1987), no. $1,51-67$.

[21] O. Labs and D. van Straten, The Cubic Surface Homepage, Tech. report, University of Mainz, 2001, www.Cubics.AlgebraicSurface.net.

[22] F. J. López, F. Martín, and S. Morales. Adding handles to Nadirashvili's surfaces. J. Differential Geom., 60(1):155-175, 2002.

[23] F. Martín and S. Morales. Complete proper minimal surfaces in convex bodies of $\mathbb{R}^{3}$. Duke Math. J., 128(3):559-593, 2005.

[24] Y. Martinez-Maure. Contre-exemple à une caractérisation conjecturée de la sphère. C. R. Acad. Sci. Paris Sér. I Math., 332(1):41-44, 2001.

[25] W. Meeks. Personal email. Apr. 6, 2006.

[26] N. Nadirashvili. Hadamard's and Calabi-Yau's conjectures on negatively curved and minimal surfaces. Invent. Math., 126(3):457-465, 1996.

[27] G. Panina. On hyperbolic virtual polytopes and hyperbolic fans. Cent. Eur. J. Math., 4(2):270293, 2006.

[28] È. R. Rozendorn. Weakly irregular surfaces of negative curvature. Uspehi Mat. Nauk, 21(5 (131)):59-116, 1966. Engl. transl.: Russ. Math. Surv. 21(5):57-112. 1966.

[29] , Surfaces of negative curvature [ MR1039819 (92a:53087)]. In Geometry, III, volume 48 of Encyclopaedia Math. Sci., pages 87-178. Springer, Berlin, 1992.

[30] G. Salmon, On the triple tangent planes to a surface of the third order, Camb. and Dublin Math. Journal 4 (1849), 252-260.

[31] L. Schläfli, On the distribution of surfaces of the third order into species in reference to the presence or absense of singular points, and the reality of their lines, Phil. Trans. Royal Soc. London 153 (1863), 193-241.

[32] B. Segre, The Non-singular Cubic Surfaces, Oxford University Press, Oxford, 1942.

[33] Steiner, Über die Flächen dritten Grades, J. Reine Angew. Math. 53 (1857), 133-141.

[34] S. Wolfram. The Mathematica ${ }^{\circledR}$ book, Fifth Edition. Wolfram Media, Inc., Champaign, IL, 2003. 
Department of Mathematics,Indiana University Bloomington, IN 47405

E-mail address: connell@indiana.edu

$U R L$ : mypage.iu.edu/ connell

School of Mathematics, Georgia Institute of Technology, Atlanta, GA 30332

E-mail address: ghomi@math.gatech.edu

$U R L$ : www . math.gatech.edu/ ghomi 